\title{
Dynamic risk simulation to assess natural hazards risk along roads
}

\author{
J. Voumard ${ }^{1}$, O. Caspar ${ }^{2}$, M.-H. Derron ${ }^{1}$, and M. Jaboyedoff ${ }^{1}$ \\ ${ }^{1}$ Research Centre on Terrestrial Environment, University of Lausanne, Lausanne, Switzerland \\ ${ }^{2}$ Touring Club Suisse, Geneva, Switzerland \\ Correspondence to: J. Voumard (jeremie.voumard@unil.ch) \\ Received: 28 February 2013 - Published in Nat. Hazards Earth Syst. Sci. Discuss.: 17 April 2013 \\ Revised: 20 September 2013 - Accepted: 3 October 2013 - Published: 5 November 2013
}

\begin{abstract}
Risk generated by natural hazards on roads is usually calculated with equations integrating various parameters related to hazard and traffic. These are static variables, like an average number of vehicles crossing this section every day and an average vehicle speed. This methodology cannot take into account dynamic variations of traffic and interactions between vehicles such as speed modifications due to windy roads, slowdowns resulting from saturated traffic or vehicle tailbacks forming in front of traffic lights.

Here we show, by means of a dynamic traffic simulator, that traffic variations may greatly influence the risk estimation over time. The risk is analysed on several sections of an Alpine road in Switzerland using a dynamic vehicles approach, and compared with the results of the static methodology. It demonstrates that risk can significantly increase on sinuous sections because of decreasing vehicle speed. For example, along an $800 \mathrm{~m}$-long section of road containing two hairpin bends, the dynamic risk is about $50 \%$ higher than the static one. Badly placed signalization, slowing down, or stopping the vehicles in a hazardous area may increase the risk by about $150 \%$ (i.e. 2.5 times higher) along a straight road section where vehicles speed is high.

A more realistic risk can thus be obtained from a dynamic approach, especially on mountain roads. The dynamic traffic simulator developed for this work appears to be a helpful tool to support decision-making in reducing risk on mountain roads and it shows the importance of keeping the traffic moving as freely as possible.
\end{abstract}

\section{Introduction}

Transportation corridors, especially in the mountains, are frequently exposed to natural hazards, such as rockfalls (Budetta, 2004; Bunce et al., 1997) or landslides (Al-Homoud, 1997; Collins, 2008; Guemache, 2011; Salcedo, 2009) according to the definitions of landslide types and processes from Cruden and Varnes (1996) and Hungr et al. (2012). Those natural hazards may threaten road users at different levels, including human, social and economic levels causing injuries, deaths, damages and delays (Bunce et al., 1997). Because it is not possible to protect every kilometre of roads threatened by mass movements, the risk for road users has to be assessed to highlight the road sections where mitigation measures are really required. If that risk is higher than the accepted risk, mitigation measures should be put in place to reduce it to an acceptable level. Assessing the risk of mass movements on a highway network is not obvious, however, because it depends on several factors such as event frequency, average vehicle speed, decision/sight distance, road characteristics and traffic on each road section (Budetta, 2004).

Some methodologies to evaluate the risk along major highways have been proposed by governmental road agencies, in Switzerland (Borter, 1999; Cajos et al., 2009; Dorren et al., 2009; FEDRO, 2009), USA (Pierson et al., 1990; Roberds, 2005) and Canada (Hungr et al., 1999). For the Alps, some research works have also been published in Baillifard et al. (2003) and Michoud et al. (2012) or within international research projects like MASSA (2010, for Medium And Small Size rock fall hazard Assessment, in Switzerland, Italy and France). Cost/benefit analyses have also been produced in the case of a road closing (Wilhelm, 1997). 
Table 1. Parameters used to assess risk from natural hazards on roads. Bold: output results and input parameters needed to solve the kinematics object risk equation. Normal: intermediary parameters solved during the calculation by the simulator. Italic: used only in equations.

\begin{tabular}{|c|c|c|}
\hline Acronym & Description & Unit \\
\hline$R_{\mathrm{ob}}$ & Object risk & {$\left[\operatorname{dead} \mathrm{yr}^{-1}\right.$ ] or $\left[\mathrm{USD} \mathrm{yr}^{-1}\right]$} \\
\hline $\boldsymbol{R}_{\text {ind }}$ & Individual risk & {$\left[\operatorname{dead} \mathbf{y r}^{-1}\right]$ or $\left[\mathrm{USD} \mathrm{yr}^{-1}\right]$} \\
\hline$F_{\mathrm{e}}$ & Occurrence frequency of an event & {$\left[\mathbf{y r}^{-1}\right]$} \\
\hline$P_{\mathrm{s}}$ & Damaged proportion of the road $[0-1]$ & {$[-]$} \\
\hline$\lambda$ & Death probability when a vehicle is touched $[0-1]$ & {$[-]$} \\
\hline$\beta$ & Average vehicle occupation & [person] \\
\hline$t_{\text {sim }}$ & Simulation time & {$[\mathrm{s}]$} \\
\hline$H$ & Hazard & {$\left[\mathrm{yr}^{-1}\right]$} \\
\hline $\operatorname{Exp}_{i}$ & Exposure & {$[-]$} \\
\hline$V$ & Vulnerability & {$[-]$} \\
\hline$W$ & Potential total loss of persons or costs & [dead] or [USD] \\
\hline$X$ & Daily number of time a person passing through the hazardous road section & {$\left[\right.$ day $\left.^{-1}\right]$} \\
\hline$t_{\text {cum }}$ & Cumulated time of vehicles observed in the hazardous area during a simulation & [nb vehicles s] \\
\hline$N_{\mathrm{v}}$ & Number of vehicles exposed in the hazardous area during a given time & [nb vehicles] \\
\hline$l$ & Length of the hazardous section & {$[\mathrm{m}]$} \\
\hline$f$ & Conversion factor to convert $\left[\mathrm{km} \mathrm{min}^{-1}\right]$ to $\left[\mathrm{m} \mathrm{day}^{-1}\right]$ & {$[-]$} \\
\hline$v$ & Mean speed & {$\left[k m h^{-1}\right]$} \\
\hline
\end{tabular}

All of these methods use so-called static traffic values to assess the risk. It means that the number of vehicles on a road section is defined by an average number of vehicles per time unit (daily or annually) and that the vehicles speed is usually the same for each of them. Generally two types of risk are calculated: (1) the object risk, which is the probability that a driver is killed among the total amount of persons passing through the hazardous area; and (2) the individual risk, which is the probability that a driver passing $\mathrm{N}$ times per day in a hazardous area is killed. This article focuses on the object risk. Parameters used in risk equations are summarized in Table 1.

In this paper, we propose an assessment of the risk that integrates dynamic variables of traffic in the calculation. The aims of this approach are: (1) to better understand the influences of vehicles' speed and traffic density on the risk results; (2) to evaluate the consequences of vehicle tailbacks induced by traffic lights or following an event like a fallen rock on the lanes. A dynamic traffic simulator was developed to simulate a part of vehicles interactions for different scenarios along a real Alpine road in Switzerland.

The main parameter required to include the traffic dynamics into the risk calculation is the duration of presence of vehicles inside the hazardous road section during a time period. It is thereby possible to take into consideration the differences between the exposition of slow vehicles which stay longer in the hazardous area and increase their risk, and the exposition of fast vehicles, which are less likely to be hit by a stone but are more likely to crash against an obstacle encountered in the road

\section{Methodology}

\subsection{Dynamic traffic simulator}

Different traffic simulators, models and methods were investigated in the literature (Barceló, 2010; Kerner, 2009; Treiber and Kesting, 2010) to be used for this risk analysis. We searched for a microscopic (i.e. each vehicle is simulated independently) dynamic traffic simulator taking into account elements degrading the traffic, able to calculate the presence of vehicles inside a definite hazardous area and including accident possibilities. A few years ago, the Intelligent Driver Model (Treiber and Kesting, 2010) or the Modern Traffic Flow Theory (Kerner, 2009) were proposed. Those microscopic models are not adapted for a dynamic traffic-riskoriented modelling because they cannot simulate accidents (Schönhof and Helbing, 2009; Treiber and Kesting, 2010) which is a requirement to work on mountain roads threatened by natural hazards. The queuing models for road traffic flows (Miller, 1960) or to analyse the performance of a congested roadway segment (Rajat et al., 1997) are interesting for the dynamic risk calculation but they are based on overtaking possibilities which are unrealistic on mountain roads. A problem in microscopic simulation is the resources needed to simulate the behaviour of each vehicle (Cameron and Duncan, 1996). This is a reason why traffic is often modelled with macroscopic models, i.e. as a kind of continuous flow. But this kind of model does not fulfil our requirements for detailed risk calculation. Indeed, traffic simulations related to natural hazard are done for emergency evacuation planning, like for example degradable transportation systems (DTS). Sensitivity and reliability analyses of these 
macroscopic-scale models are described by Du and Nicholson (1997). Socio-economic impacts of a natural hazard like hurricanes can also be measured with DTS in order to minimize losses (Nicholson and Du, 1997). Jha et al. (2004) propose evacuation plans based on a microscopic dynamic traffic model called MITSIMLab (Yang et al., 2000; Moshe et al., 2010) while contra-flow strategies for a large-scale regional traffic are simulated by Chiu et al. (2008).

However interesting they may be, these models do not fit the requirements of a simulator specific to dynamic traffic on mountain roads. Therefore, it was decided to develop our own dynamic traffic simulator, within the numerical computing environment of MATLAB ${ }^{\circledR}$.

In this model, a road is composed of two lanes, one for each direction, and vehicles can only drive on their own lane. There is no possibility for a vehicle to overtake or to move from one lane to the other. Variables are declared in the box A (Fig. 1) and are followed by a time loop where two vehicles loops are integrated, one per lane. After calculating positions of all vehicles in lane 1 at time $t$ (Fig. 1b), the simulator calculates positions of all vehicles on lane 2 at the same time (Fig. 1c). After computing positions of all vehicles at time $t$, the number of vehicles in the hazardous area is counted and stored for further calculation. Then the next time step is simulated with a typical increment of one second. Finally, the cumulated time of vehicles observed in the hazardous area during a simulation is calculated and graphs are produced (Fig. 1d). After completing a simulation, the dynamic and static risks are calculated (Fig. 1e).

Vehicles have initial speeds which are a combination of the maximum authorised speed on the section added with a random variation margin specific to each vehicle. On a lane, the first car adapts its speed in function of the road sinuosity and obstacles on the road like traffic lights. The following vehicles fit their speed (again with the two components: maximal authorized speed and random margin) with the road sinuosity, possible obstacles and the distance to the previous vehicle.

Three mechanisms govern the vehicles kinematics: one acceleration and two types of decelerations (one light, taking the foot off the accelerator and one strong, using the brake). A vehicle tends to accelerate if its speed is lower than the maximal authorised speed, if the sinuosity is low and if the distance to the previous vehicle is long enough or if the previous vehicle drives faster and if there is no obstacle on the road. A vehicle brakes in function of the visibility distance, the sinuosity of the section, the distance to the previous vehicle and the presence of an obstacle on the road. Thus, the vehicle speed depends on the traffic density, the road geometry (2-D), the vehicle in front's speed and the presence of obstacles on the road section (Appendix A).

All of the kinematic parameters as well as the visibility distance (not depending on topography, only on sinuosity) and speed parameters (with a total of 60 parameters) can be

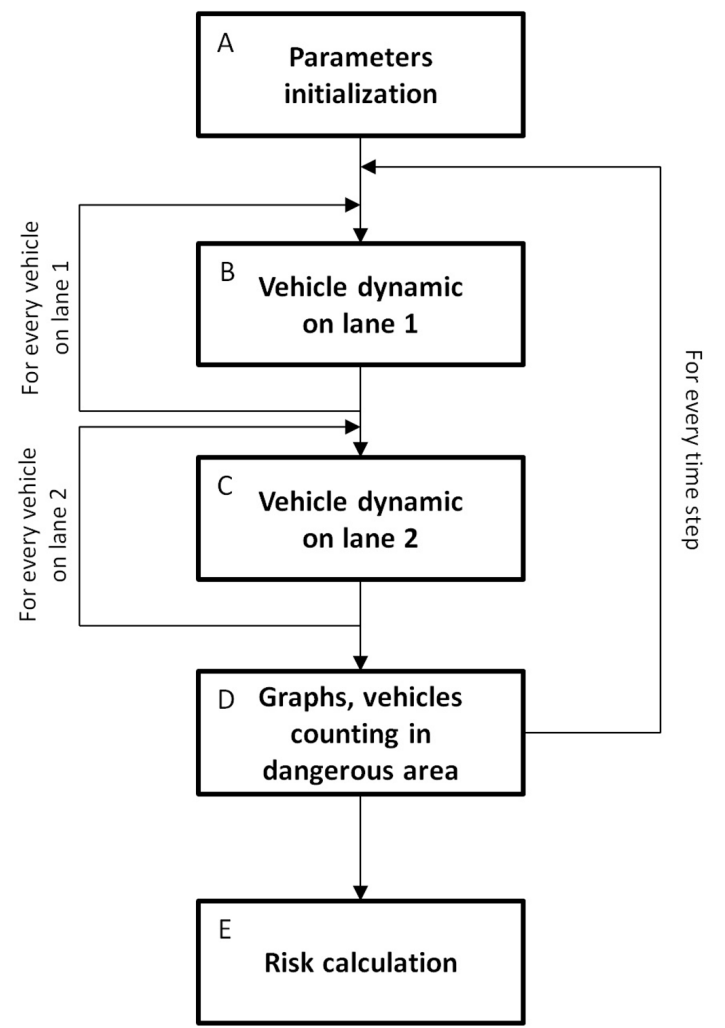

Fig. 1. Structure of the traffic simulator model. Box A is read once to initialize the parameters and the first vehicle on the road. Boxes $\mathrm{B}$ and $\mathrm{C}$ belong to the temporal loop which increments time every time step. During one time unit (1 s), all the vehicles' positions on lane 1 (box B) and on lane 2 (box C) are calculated. Finally, after the traffic simulation, different factors related to the risk calculation are computed in boxes D and $\mathrm{E}$.

defined directly in the graphic user interface (GUI) (Fig. 2) or in input files.

A lane is constructed as a suite of nodes connected by segments. Segments do not have to be the same length, so that nodes can be chosen according to the roads geometry. Vehicles follow the curvilinear abscissa of the lane on which they are.

\subsection{Static risk calculation}

To compare the risk from the dynamic traffic simulator with the usual static approach, the static risk was calculated based on the common risk equation (Einstein, 1988; Fell et al., 2005):

$R=\sum_{i=1}^{n} H \cdot \operatorname{Exp}_{i} \cdot V \cdot W$,

where $R$ is the risk [dead $\mathrm{yr}^{-1}$ ] or [USD yr ${ }^{-1}$ ] with $n$ objects, $H$ is the hazard $\left[\mathrm{yr}^{-1}\right], \operatorname{Exp}_{i}$ is the object exposure, i.e. the probability that a vehicle is hit in the hazardous area $[-], V$ 


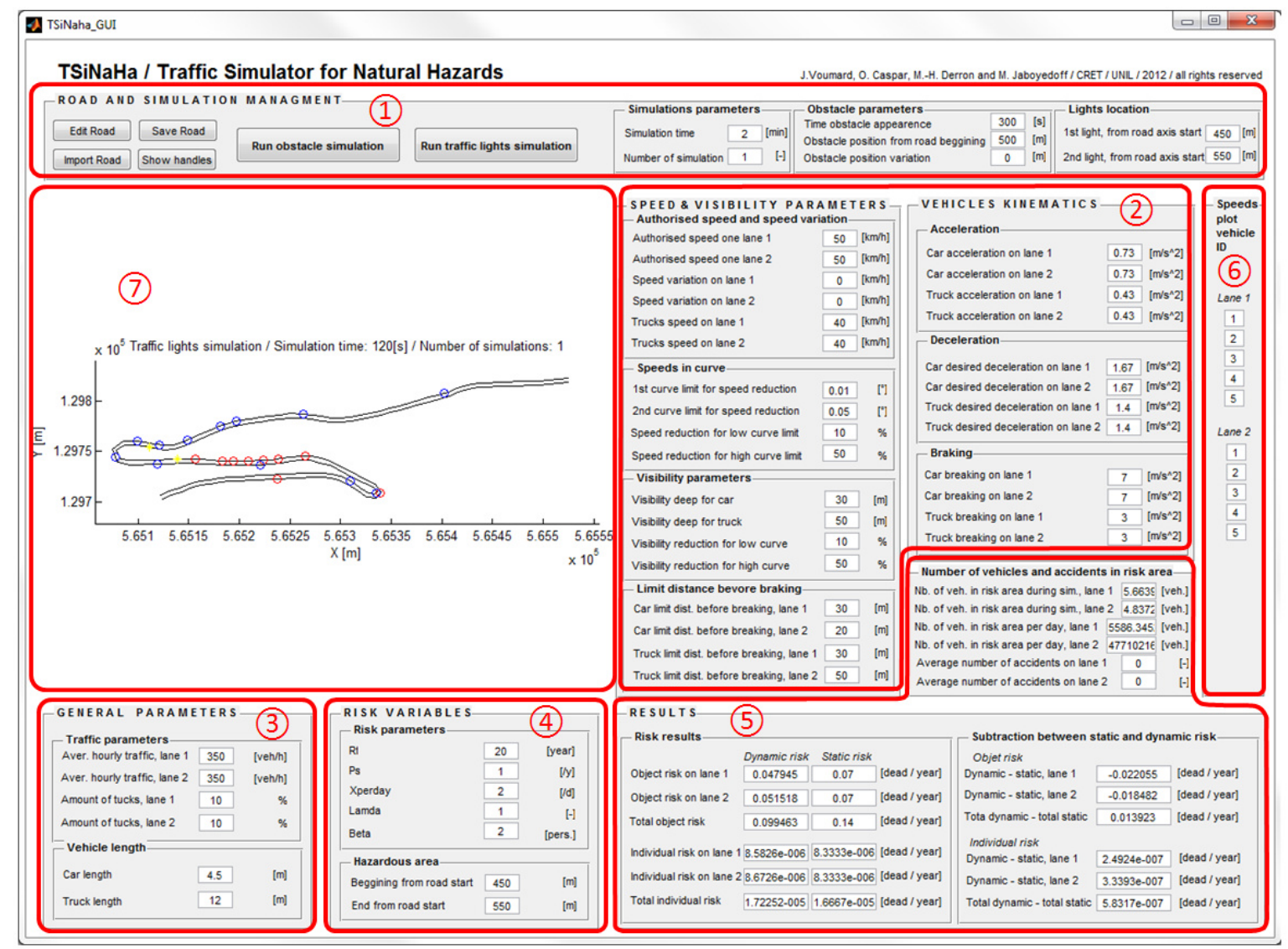

Fig. 2. Graphic user interface (GUI) of the dynamic traffic simulator in MATLAB ${ }^{\circledR}$. The GUI is divide into seven boxes: (1) simulation and road management importation of a road section to choose scenario type and to define traffic lights and obstacle location as well as simulation time; (2) speed and visibility parameters, introduction of the different parameters of vehicle's kinematics (acceleration, breaking), speed limit and speed reduction in curve linked with visibility parameters; (3) general traffic parameters and vehicle lengths; (4) start and end of the hazardous area location and static parameters used for the risk equation; (5) results of the simulation appear here with number of vehicles in the hazardous area, number of accidents and dynamic and static risk results and comparison; (6) vehicle identifiers to be plotted in the speed graphic; (7) graph of the road section, the hazardous area, traffic lights and obstacle locations and vehicles' displacement.

is the object vulnerability [-] and $W$ is the potential total loss of persons or costs ([dead] or USD]).

Fell et al. (2005) and Bründl (2009) have modified Eq. (1) to calculate the object risk on a road:

$R_{\mathrm{ob}}=F_{\mathrm{e}} \cdot P_{\mathrm{s}} \cdot N_{\mathrm{v}} \cdot \lambda \cdot \beta$,

where $R_{\mathrm{ob}}$ is the object risk [dead $\mathrm{yr}^{-1}$ ], $F_{\mathrm{e}}$ is the occurrence frequency of an event $\left[\mathrm{yr}^{-1}\right], P_{\mathrm{s}}$ is the proportion of the hazardous section which is affected when a hazard occurs $[-], \lambda$ is the death probability when a vehicle is damaged by a hazard [-], $\beta$ the average vehicle occupation [person/vehicle], and $N_{\mathrm{v}}$ is the number of equivalent vehicles permanently exposed in the hazardous area [vehicles nb.]:

$N_{\mathrm{v}}=\frac{N_{\mathrm{v} \_ \text {tot }}}{f} \cdot \frac{l}{v}$,

where $N_{\mathrm{v}_{-} \text {tot }}$ is average number of vehicles per day [vehicles nb day $\left.{ }^{-1}\right], l$ is the length of the hazardous section [m], $v$ is the average vehicle speed $\left[\mathrm{km} \mathrm{h}^{-1}\right]$ and $f$ is a conversion factor to convert the speed from $\left[\mathrm{km} \mathrm{h}^{-1}\right]$ to $\mathrm{m} \mathrm{day}^{-1}$. Comparing Eqs. (1) and (2), $F_{\mathrm{e}}$ and $P_{\mathrm{s}}$ represent $H$, where $P_{\mathrm{s}}$ allows the hazard on a road section to spread. $N_{\mathrm{v}}$ is the sum of exposures $\left(\operatorname{Exp}_{i}\right), \lambda$ the vulnerability $V$, and $\beta$ is the losses $W$.

The common equation to estimate the individual risk is

$R_{\text {ind }}=\frac{R_{\mathrm{ob}} \cdot X}{N_{\mathrm{v}_{\mathrm{t}} \mathrm{tot}} \cdot \beta}$,

where $X$ is the amount of time that a person passes every day through the hazardous road section $\left[\right.$ day $\left.^{-1}\right]$.

\subsection{Dynamic risk calculation}

The main concept to calculate risk for dynamic traffic is to measure the duration of presence of vehicles inside the hazardous area during a given time. This way slow vehicles, which stay longer in the hazardous area, are more exposed by 


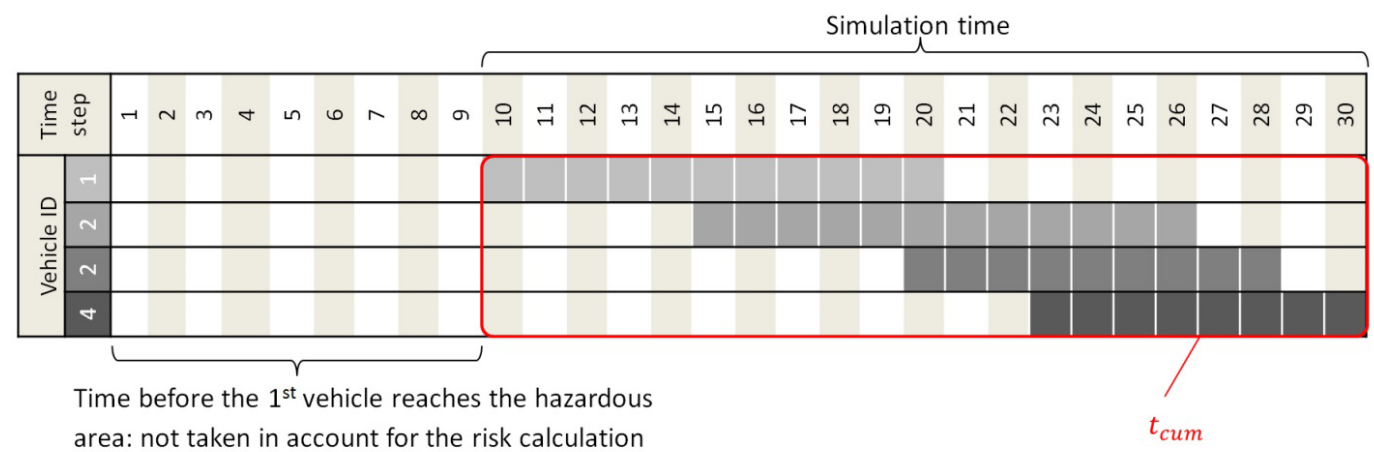

Fig. 3. Cumulated time of vehicles observed in the hazardous area during one simulation. In this example, four vehicles passed through the hazardous area with a total of 40 time steps of cumulated time. Here, one time step is equal to one second. During the first $9 \mathrm{~s}$ of the simulation, no vehicle has reached the hazardous area. Thus, time required by the first vehicle to reach the hazardous zone is not included in the simulation duration.

a hazard. Thus, each vehicle presence is analysed and recognised. The simulator counts the presence of vehicles in the hazardous area by looking every second if vehicles are located in the section. It measures the cumulated time of vehicles observed in the hazardous area during a simulation time, $t_{\text {cum }}$, in function of time steps, number of vehicles and section number (Fig. 3):

$t_{\text {cum }}=\sum_{i=1}^{t_{\text {sim }}} \sum_{j=1}^{n} i f\left(x\left(t_{i}\right)_{j} \in D_{k}\right) \Delta t$,

where $D_{k}$ is the domain of $k$ sections and $\Delta t=x\left(t_{i+1}\right)-$ $x\left(t_{i}\right)$ is constant, $i$ is the time index, $j$ the vehicle index and $n$ the number of vehicles generated during the simulation.

When the simulation starts, there are no vehicles on the road (initial condition). The simulation time, $t_{\text {sim }}$, starts only when the first vehicle enters in the hazardous section. At the end of the simulation, the cumulated time of vehicles observed in the hazardous area during a simulation, $t_{\text {cum }}$, is divided by the duration of the simulation:

$N_{\mathrm{v}}=\frac{t_{\text {cum }}}{t_{\text {sim }}}$.

Thus, we obtain the equivalent number of vehicles exposed permanently in the hazardous area. For example, if we obtain a cumulated time of vehicles in the hazardous area of $120 \mathrm{~s}$ during a $60 \mathrm{~s}$ simulation, this is equivalent to two vehicles which are permanently in the hazardous area during the $60 \mathrm{~s}$ of simulation time. But it may also be 12 vehicles passing through the area during $10 \mathrm{~s}$ or six vehicles which stay $20 \mathrm{~s}$ in the hazardous section. It is not important to know how long each vehicle stays in the hazardous section but it is necessary to know the total exposure of vehicles in this section.

Therefore, based on Eq. (2), the dynamic object risk is

$R_{\mathrm{ob}}=F_{\mathrm{e}} \cdot P_{\mathrm{s}} \cdot N_{\mathrm{v}} \cdot \lambda \cdot \beta=F_{\mathrm{e}} \cdot P_{\mathrm{s}} \cdot \frac{t_{\mathrm{cum}}}{t_{\mathrm{sim}}} \cdot \lambda \cdot \beta$.
With this approach, the vehicles velocities are removed from the equation and only the vehicles in the hazardous area are counted. This enfranchisement is fundamental for the calculation of dynamic risk. In this way, all vehicles can have their own speed and the risk depends only on the actual cumulated staying time of vehicles in the hazardous area.

\section{Case study}

\subsection{Description and location of case study}

Eight road sections threatened by different natural hazards like rockfalls, debris flows or dolines have been studied along the mountain road Aigle - Col du Pillon in western Switzerland. Three of these sections are presented in this paper (Fig. 4 and Table 2):

1. Fontanney section, with two hairpin bends, is crossed by a pressure pipe which could generate debris flows in case of rupture. The major danger is that such an event might destroy a vehicle tailback waiting in front of a traffic light.

2. Pont-Bourquin section is threatened by an active landslide. The road forms a long curve located at the base of the landslide.

3. Col du Pillon section is located on a gypseous basement where dolines can form. In 2009, a doline destroyed a portion of the road.

\subsection{Scenarios}

Two different scenarios were simulated on the different road sections: (1) a road without any obstacles and (2) a road regulated by traffic lights. Each road section has two lanes, one uphill and one downhill. Each simulation lasts ten minutes; 

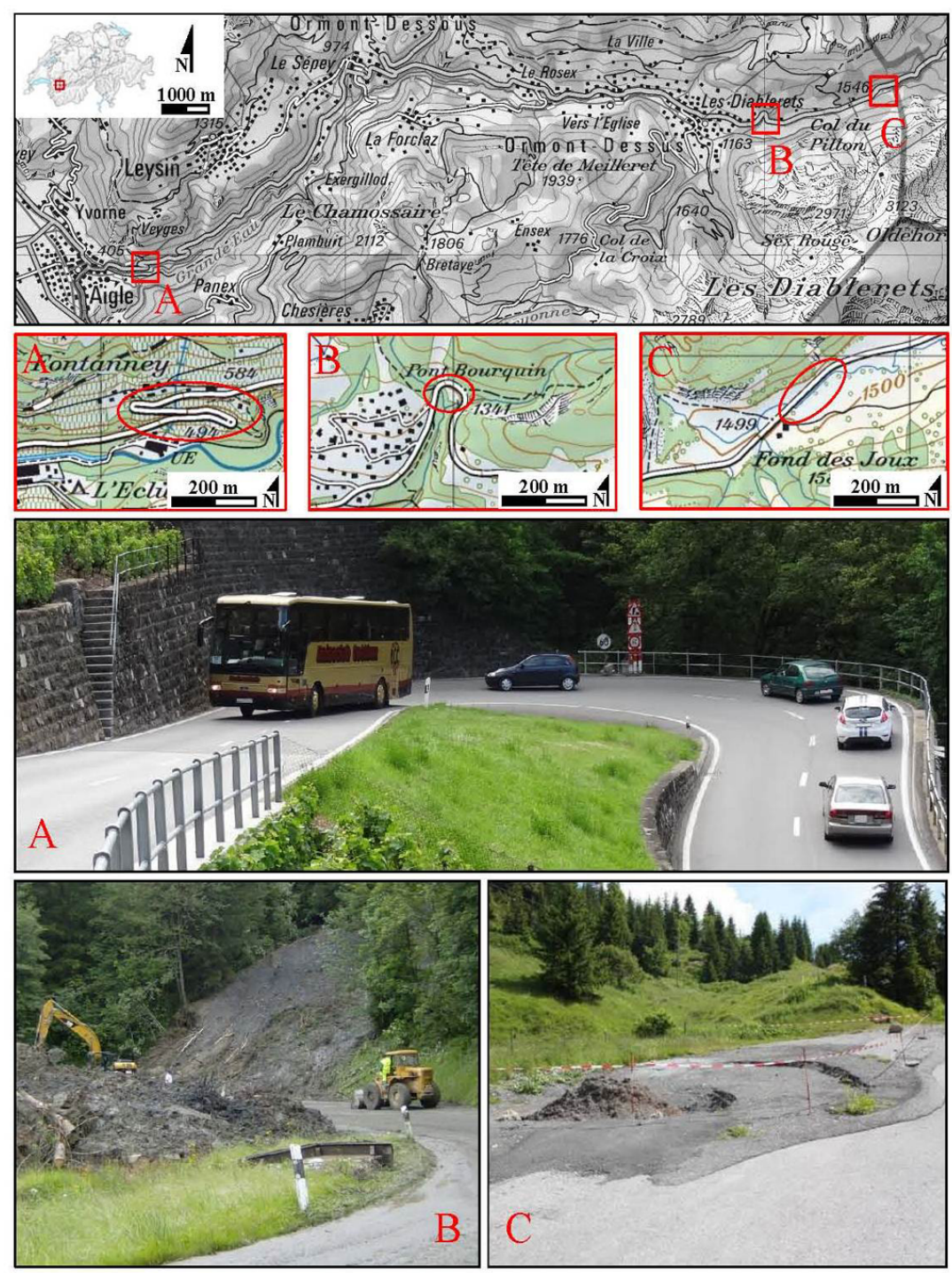

Fig. 4. Location of the three sections on the study road Aigle - Col du Pillon, in Vaud canton, Switzerland. (A) Fontanney section with debris flows hazard. The picture shows the south hairpin bend with road works traffic signs and different types of vehicles (2012). (B) PontBourquin section threatened by an active landslide (picture of the 2007 event). (C) Col du Pillon section with doline hazard (picture of the 2009 event). (Copyright for topographical maps: Swisstopo).

Table 2. Description of the three sections from the studied road Aigle - Col du Pillon, in Vaud canton, Switzerland. The results of this simulation section are describe bellow. The average speed of vehicles was measured in the field using a pocket traffic radar.

\begin{tabular}{|c|c|c|c|}
\hline Section & Description & Natural hazard & $\begin{array}{l}\text { Average vehicle speed } \\
\text { measured in the field } \\
{\left[\mathrm{km} \mathrm{h}^{-1}\right]}\end{array}$ \\
\hline Fontanney & $\begin{array}{l}\text { S-track with } 2 \text { hairpin bends } \\
\text { crossed by a pressure pipe }\end{array}$ & $\begin{array}{l}\text { Debris flows in case of failure } \\
\text { of the pressure pipe }\end{array}$ & 45 \\
\hline Pont-Bourquin & Large hairpin bend & Active landslide beside the road & 35 \\
\hline Col du Pillon & Straight line & Doline & 85 \\
\hline
\end{tabular}


the risk is scaled to one year in the risk calculation. The scenarios are fictive but the three sectors match the dangerous areas and frequent engineering works.

In the first scenario (Fig. 5a), the road configuration is in a normal operational state, without any obstruction or traffic regulation. The only obstacle to the circulation is a high density of vehicles creating a slowdown. This scenario allows for the comparison of dynamic risk calculation with the standard static method. It is indeed useful for sections with many turns to observe the slowdown of vehicles in curves, which influences vehicle presence in a hazardous area that impacts the risk. Another effect on the traffic is the different types of vehicles driving on the road, like trucks generating tailbacks of cars.

The second scenario (Fig. 5b) represents the presence of traffic lights on the road. This traffic regulation is often encountered on mountain roads because of the numerous road works and maintenance sites. Traffic lights can be positioned outside the hazardous area, can overlap it or can be placed inside it. It is obvious that the risk increases when vehicles are stopped in front of traffic lights located in the hazardous area. Traffic lights in the Fontanney section are located inside the hazardous area where vehicle tailbacks in the hazardous section could reach $400 \mathrm{~m}$-long for lane 1 and $200 \mathrm{~m}$-long for lane 2. Traffic lights in the Pont-Bourquin section are positioned outside the hazardous area because an early warning system has been installed to prevent vehicles crossing the landslide area during an event. It means there is no vehicle tailback in the hazardous area. Finally, the traffic lights of the Col du Pillon section are located inside (lane 1) and outside (lane 2) the hazardous area. It means that a $100 \mathrm{~m}$-long vehicle tailback can form in the lane 1 in the hazardous area. The traffic light in lane 1 is first green during $1 \mathrm{~min}$ while the light on lane 2 is red. From $60 \mathrm{~s}$ to $70 \mathrm{~s}$, both lights are red and then light on lane 2 switches to green.

\subsection{Numerical setups}

Parameters of the simulator have been defined based on data from the literature and on site measurements. Usual settings and traffic parameters were selected based on data from the Roads Office of the Vaud canton (2012), the largest federated state of the French-speaking part of Switzerland, and the FEDRO (2012), the Federal Roads Office of Switzerland. A regular counting of vehicles on the road provides precise data of the traffic classes (vehicle types) running on the section and gives hourly variations of the flux. The average flux of traffic is defined as 250 vehicles per hour for the Fontanney section, 65 vehicles per hour for the Pont-Bourquin section and 30 vehicles per hour for the Pillon section, corresponding to the maximal daily traffic (ADT) on those sections. The length of cars was determined from average lengths obtained from internet websites of car manufacturers $(4.5 \mathrm{~m})$. The length chosen for trucks $(12 \mathrm{~m})$ is an approximation of the length of the majority of trucks observed during the field measurements although much longer road trains were observed.

Speed and visibility variables are based on field observations. They differ according to the road sections. In Switzerland, the speed limit on roads outside localities is $80 \mathrm{~km} \mathrm{~h}^{-1}$. Around temporary traffic lights site construction, the limit is reduced to $60 \mathrm{~km} \mathrm{~h}^{-1}$ on the studied road. The speed reductions in curves were calibrated using measurements made in the field of vehicle speed in curves. Achieved truck speed was set from 30 to $50 \mathrm{~km} \mathrm{~h}^{-1}$, according to the sections; speeds they rarely exceed on a mountain road. Visibility distances also come from field observations. Truck drivers have a better visibility than car drivers because of their higher position above the road.

Distance limits before the vehicles brakes are estimated from field observations by estimating the minimum distance before a vehicle brakes. They adapt their speed regarding the lower speed of the previous vehicle or stopping in front of an obstacle. These parameters were chosen in coherence with field observations and maintained constant for the different simulations. Thus, it was possible to compare the scenarios and the different road segments. Some parameters of the simulations are presented in Table 3.

Parameters used in the simulator can be evaluated by three different ways (Tables 4 and 5). The first and the best way to collect data is in the field with a pocket radar (which can measure vehicle speed very easily) and an EDM (electronic distance measurement; unit to evaluate different distances on the road). Except speed, the second way to get parameter values is to measure them on a satellite image or airphotos, e.g. Google Maps ${ }^{\circledR}$ or a local geoportal. Finally, the third way is to collect data from the literature, especially for vehicle kinematic and traffic values like ADT (average daily traffic). That can be from books about transportation (Khisty and Lall, 2003; McShane et al., 1998) or from local reports about a specific road section (ASTRA, 2012; Canton de Vaud, 2012). Traffic information and data can also be obtained from people working on or knowing well the studied road section.

When it is not possible to go to the study area, speeds and distance values must be obtained with the help of the literature and of people who have experience in the local traffic. Knowing the geometry (length of straight lines, curves radius, width of the road section a sometimes even the slope) of a road section is usually enough to evaluate roughly the speed and visibility parameters. These values will be less accurate than values obtained on site but, from experience, good enough to run simulations and to get a rough estimate of the risk for a hazardous area. More difficult is to obtain acceleration and deceleration properties. This comes especially from the fact that kinematics is always different regarding the function of the road geometry and conditions, and the vehicle characteristics. Parameters for an Alpine Swiss road cannot be used for trucks in Nepal. Some typical vehicle kinematic parameters can be found in Li and Duon (2011), Peng et al. (2011) and Yu et al. (2013). 


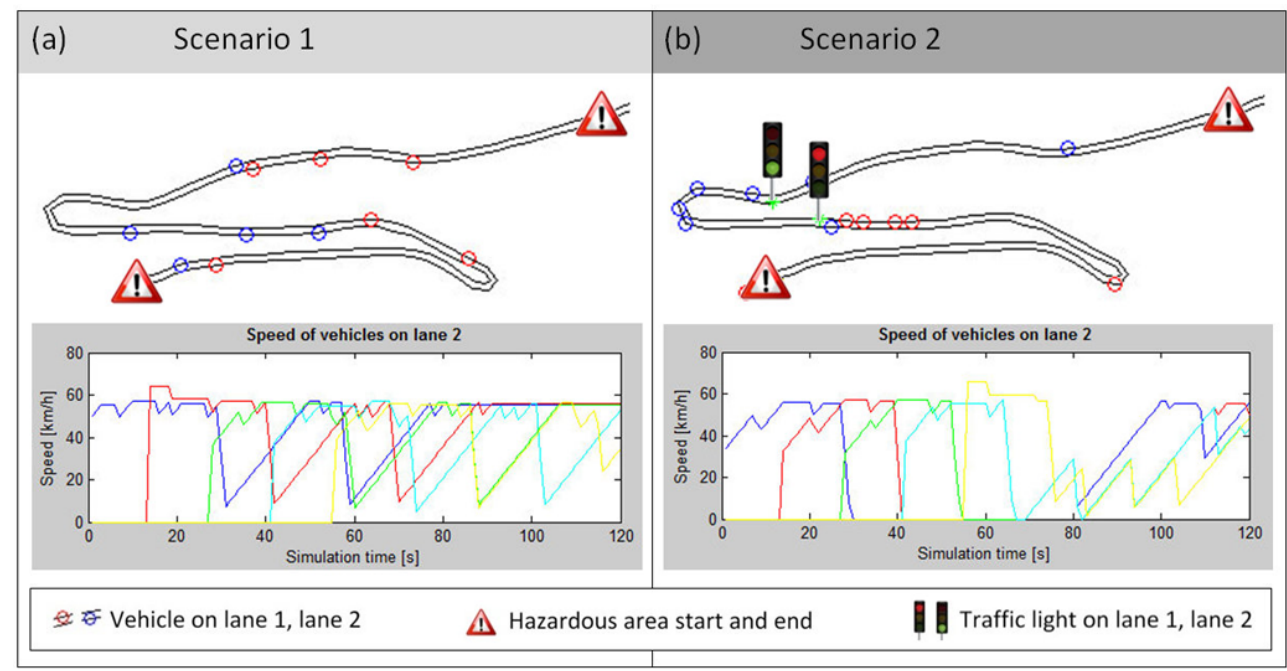

Fig. 5. Illustration of the three scenarios on the Fontanney section with corresponding speed graphics of lane 2 . The caution panels indicate the beginning and the end of the hazardous area. Vehicles are represented by circles, red for the lane 1 (uphill) and blue for the lane 2 (downhill). (a) Scenario 1: road without obstacles or traffic limitations. Vehicle speeds vary only in function of the road geometry. (b) Scenario 2: traffic regulated by traffic lights. A vehicle tailback forms behind the red traffic light (lane 2) while the vehicles on lane 1 cross the hazardous area. Vehicle speeds fall down to zero $\mathrm{km} \mathrm{h}^{-1}$ during $20 \mathrm{~s}$ before increasing after the traffic light turns to green.

Table 3. Parameters for the simulations.

\begin{tabular}{|c|c|c|c|c|}
\hline \multirow[t]{2}{*}{ Parameter } & \multirow[t]{2}{*}{ Unit } & \multicolumn{3}{|c|}{ Section } \\
\hline & & Fontanney & Pont-Bourquin & Pillon \\
\hline Section length & {$[\mathrm{m}]$} & 3500 & 2800 & 2200 \\
\hline Hazardous area position & {$[\mathrm{m}]$} & $1000-1800$ & $1370-1470$ & $900-1100$ \\
\hline Hazardous area length & {$[\mathrm{m}]$} & 800 & 100 & 200 \\
\hline Damaged proportion of the hazardous area, $P_{\mathrm{S}}$ & {$[-]$} & 0.1 & 0.5 & 0.25 \\
\hline Traffic lights position & {$[\mathrm{m}]$} & $1400 \& 1600$ & $1300 \& 1500$ & $1000 \& 1100$ \\
\hline Obstacle position & {$[\mathrm{m}]$} & 500 & 1420 & 1000 \\
\hline Vehicle speed without speed reduction in curves & {$\left[\mathrm{km} \mathrm{h}^{-1}\right]$} & 70 & 50 & 80 \\
\hline Random speed variation & {$\left[\mathrm{km} \mathrm{h}^{-1}\right]$} & 30 & 20 & 30 \\
\hline Traffic density & [vehicles $\mathrm{h}^{-1}$ ] & 250 & 65 & 40 \\
\hline Return period of the hazard & {$\left[\mathrm{yr}^{-1}\right]$} & 20 & 20 & 20 \\
\hline
\end{tabular}

\section{Results}

The results of three sections of the cantonal road Aigle - Col du Pillon that were analyzed are presented below (Figs. 6 and 7, Table 6 and Appendix B). It is necessary to mention that the results differ greatly regarding the input of some parameters of the simulation. The number of vehicles per hour and the average speed are parameters that influence directly the static and dynamic risk, as well as the delimitation of the hazardous zone. Shifting the area before or after a turn can drastically change the outcome of risk. For example, in the case of dense traffic, vehicles before a tight turn will slow down sharply but go faster once they have passed it. If the hazardous area begins some tens of meters before the turn, the result of the dynamic risk will be significantly higher than a calculation taking into account only the section where the vehicles have passed the turn and drive faster. The simulator calculates results for each lane. The risk along a road section is the sum of the risk in both lanes.

The results of scenario 1 (free road) demonstrate that risk can significantly increase on sinuous sections because of decreasing vehicle speed compared to results of static risk calculation. There are two possibilities for risk increase in scenario 2 for road sections regulated by traffic lights: (a) a traffic light is placed inside the hazardous area and generates vehicle tailback in this area; the risk can increase by about $200 \%$ per lane because vehicles remain in the hazardous zone during the red light; (b) a traffic light is placed outside of the hazardous area and thereby vehicle tailback on this lane is outside of the hazardous area. For this last case, the risk increase is bigger than in scenario 1 (free road) but lower than the previous case of scenario 2 . 
Table 4. Parameter values of speed, visibility and vehicle kinematics of the simulations described in this paper. For each parameter, an evaluation scale is provided with three degrees depending of three types of evaluations methods. The degrees are: easy (fluent to obtain the value), medium (need some time to estimate the value) and difficult (theoretically possible to evaluate the value but almost impossible in reality). The "-" character means that there is no way to obtain a value using the corresponding method. The three methods of evaluations are: (1) on-site measurement (using a measuring device like speed traffic radar or electronic distance measurement [EDM]), (2) imagery (with airphotos, satellite images), and (3) literature (including traffic data from road offices, research literature and local experts knowledge [LEK]). DoRS means depend on road section: different values are given for each section.

\begin{tabular}{|c|c|c|c|c|c|c|}
\hline \multirow{2}{*}{ Parameter } & \multicolumn{2}{|c|}{ Value } & \multirow{2}{*}{ Unit } & \multicolumn{2}{|c|}{ Evaluation } & \multirow[b]{2}{*}{ Literature } \\
\hline & Car & Truck & & On-site measurement & Satellite imagery & \\
\hline Speed & \multicolumn{2}{|c|}{ DoRS } & $\mathrm{km} \mathrm{h}^{-1}$ & Easy (radar) & - & $L E K$ \\
\hline Speed variation for each vehicle & \multicolumn{2}{|c|}{ DoRS } & $\mathrm{km} \mathrm{h}^{-1}$ & Easy (radar) & - & $L E K$ \\
\hline 1 st and 2 nd curve limits for speed reduction & \multicolumn{2}{|c|}{0.001} & $\circ$ & Easy (radar) & Medium & - \\
\hline 2nd curve limit for speed reduction & \multicolumn{2}{|c|}{0.02} & $\circ$ & Easy (radar) & Medium & - \\
\hline Speed reduction for low curve & \multicolumn{2}{|c|}{20} & $\%$ & Easy (radar) & Medium & - \\
\hline Speed reduction for high curve & \multicolumn{2}{|c|}{50} & $\%$ & Easy (radar) & Medium & - \\
\hline Visibility distance [nb. of nodes] & 5 & 6 & - & Medium (EDM) & Easy & - \\
\hline Visibility reduction for low curve & \multicolumn{2}{|c|}{10} & $\%$ & Medium (EDM) & Medium & - \\
\hline Visibility reduction for high curve & \multicolumn{2}{|c|}{50} & $\%$ & Medium (EDM) & Medium & - \\
\hline Limit distance before braking & \multicolumn{2}{|c|}{25} & $\mathrm{~m}$ & Medium (observ.) & Difficult & - \\
\hline Acceleration & 0.73 & 0.43 & $\mathrm{~ms}^{-2}$ & Difficult & - & Difficult \\
\hline Deceleration & 1.67 & 1.40 & $\mathrm{~ms}^{-2}$ & Difficult & - & Difficult \\
\hline Braking & 7.00 & 3.00 & $\mathrm{~ms}^{-2}$ & Difficult & - & Difficult \\
\hline
\end{tabular}

Table 5. Risk and traffic parameters values of the simulations described in this paper. For each parameter, an evaluation scale is estimated with three degrees depending of three different methods of evaluations (see Table 4 for details).

\begin{tabular}{|c|c|c|c|c|c|}
\hline \multirow{2}{*}{ Parameter } & \multirow{2}{*}{ Symbol } & \multirow{2}{*}{ Unit } & \multicolumn{3}{|c|}{ Evaluation } \\
\hline & & & On field & Satellite imagery & Literature \\
\hline Occurrence frequency of an event & $F_{\mathrm{e}}$ & {$\left[\mathrm{yr}^{-1}\right]$} & Difficult & - & $L E K$ \\
\hline Damaged proportion of the road [0-1] & $P_{\mathrm{S}}$ & {$[-]$} & Medium & - & $L E K$ \\
\hline $\begin{array}{l}\text { Daily number of time a person passes } \\
\text { through the hazardous road section }\end{array}$ & $X$ & {$\left[\right.$ day $\left.^{-1}\right]$} & Difficult & - & $L E K$ \\
\hline $\begin{array}{l}\text { Death probability when a vehicle is af- } \\
\text { fected }\end{array}$ & $\lambda$ & {$[-]$} & Difficult & - & $L E K$ \\
\hline Average vehicle occupation & $\beta$ & [person veh. ${ }^{-1}$ ] & Medium & - & $L E K$ \\
\hline Average hourly traffic & & {$\left[\right.$ veh. $\left.h^{-1}\right]$} & Easy & - & $L E K$ \\
\hline Amount of trucks & & $\%$ & Easy & - & $L E K$ \\
\hline Vehicle length & & {$[\mathrm{m}]$} & Easy & Medium & Easy \\
\hline
\end{tabular}

Table 6. Dynamic risk results of three scenarios on the road sections and static results of those sections (sum of both lanes).

\begin{tabular}{lrrr}
\hline Section & $\begin{array}{c}\text { Static risk } \\
{\left[\text { dead }^{-1}{ }^{-1}\right]}\end{array}$ & \multicolumn{2}{c}{\begin{tabular}{c} 
Dynamic risk \\
\cline { 3 - 4 }$\left[\right.$ dead yr $^{-1}$ ]
\end{tabular}} \\
\hline Fontanney & 0.057 & 0.083 & 0.145 \\
Pont-Bourquin & 0.013 & 0.022 & 0.025 \\
Pillon & 0.005 & 0.006 & 0.011 \\
\hline
\end{tabular}

\section{Discussion}

\subsection{Comparison between static and dynamic methodologies}

Results have to be carefully analyzed to compare the static with the dynamic risk assessment methods. For the free road (scenario 1) the dynamic risk on both lanes can be up to $71 \%$ higher than the static risk for the same road section. For the Fontanney section (Table B1, Figs. 5 and B1) the dynamic risk is $45 \%$ higher than the static one. The simulated vehicles speed in the hairpin bend is very low, around $10-20 \mathrm{~km} \mathrm{~h}^{-1}$ (Fig. 8), generating a long presence in the hazardous area 


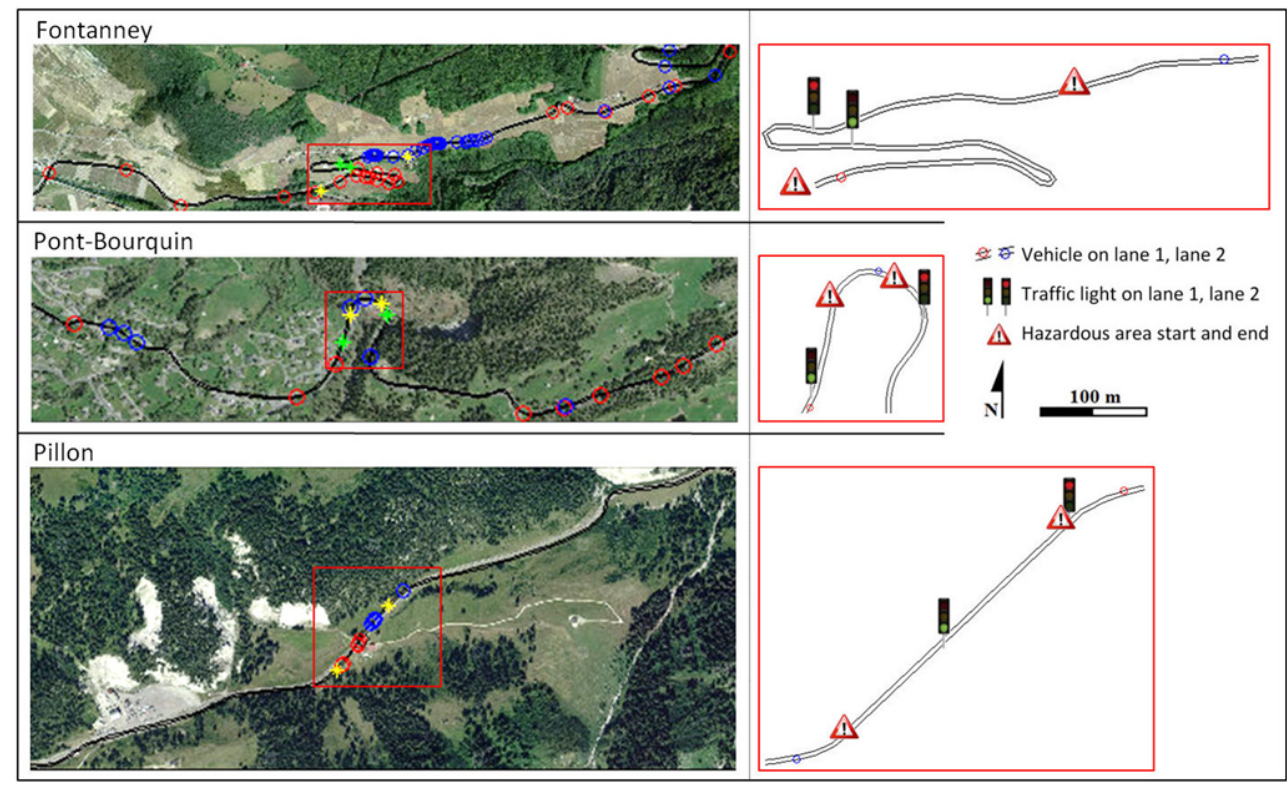

Fig. 6. The airphotos show the road sections where the traffic is simulated. These long sections are necessary to reproduce vehicles interactions similar to real ones. The images on the right are blow-ups of the hazardous area. The caution panels indicate the beginning and the end of the hazardous area; vehicles are represented by circles; traffic lights are represented with the green and red traffic lights; the yellow and green stars on the airphotos represent the limits of the hazardous area and the traffic light locations respectively. (Copyright for airphotos: Swisstopo).

which explains this risk increase. For the Pont-Bourquin section (Table B2 and Fig. B2), the dynamic risk on both lanes is about $70 \%$ higher than the static one because of the low speed in the big turn. For the Col du Pillon section (Table B3 and Fig. B3), the road is a long straight line. Most of the vehicles drive at the speed limit but some vehicles (trucks for instance) drive at a lower speed. Along this section the vehicles are not slowed down by the road geometry but only by a potentially slow moving vehicle which would slow down the traffic and form a tailback. In this case, the dynamic risk for both lanes is only $25 \%$ higher than the static one. Relative risk increase is higher in the Pont-Bourquin section than in the Fontanney section because the Pont-Bourquin hazardous area is only one big curve where vehicle speed is low while the Fontanney area contains two hairpin bends but also straight lines where vehicles drive faster.

For the second scenario, with traffic lights, we observed an increase in risk of about $155 \%$ (i.e. 2.5 times higher) for the Fontanney section compared to the static risk calculation. This is explained by the fact that the vehicles are stopped by traffic lights located in the hazardous zone. Positions of the hazardous zone and traffic lights defined in the simulation generate vehicle tailbacks of $400 \mathrm{~m}$ and $200 \mathrm{~m}$ for lane 1 and lane 2 respectively inside the hazardous area. The difference of risk increase on each lane is clear: $182 \%$ for lane 1 and $128 \%$ for lane 2 where vehicle tailback in the hazardous area is shorter than on lane 1. The Pillon section simulations show a risk increase of $130 \%$ for both lanes with a

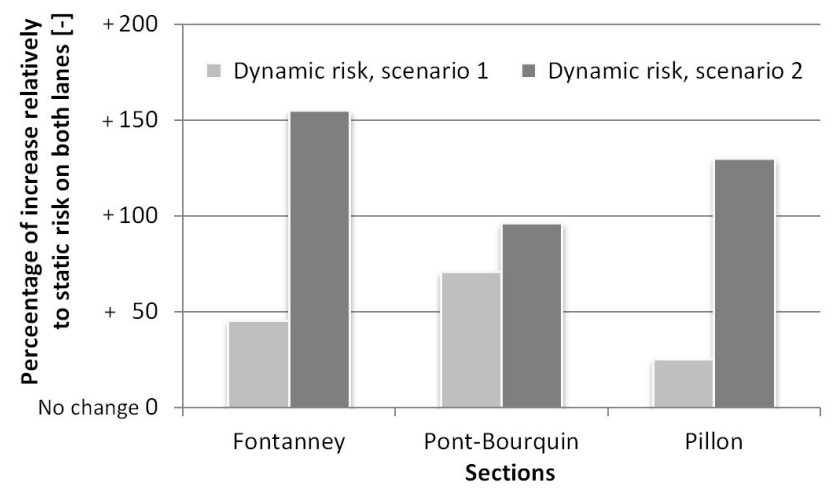

Fig. 7. Calculated dynamic risks normalized to static risk for the two scenarios (Fig. 5). Scenario 1: free road; scenario 2: traffic lights. For example, a value of about $100 \%$ for the Pont-Bourquin section with scenario 2 means that the dynamic risk for this section is nearly 2 times higher than the static risk on the same section.

strong difference between the lanes. Risk increases by $200 \%$ in lane 1 compared to static results, because the vehicles are stopped in front of the traffic light in the hazardous area on a $100 \mathrm{~m}$-long distance. In lane 2 , the risk increases only by $60 \%$ because vehicles wait outside of the hazardous area. The risk increases on lane 2 because vehicles drive through the hazardous area at a lower speed than the maximal speed of scenario 1 (free road). For the Pont Bourquin road section the dynamic risk of scenario 2 is double the static one 


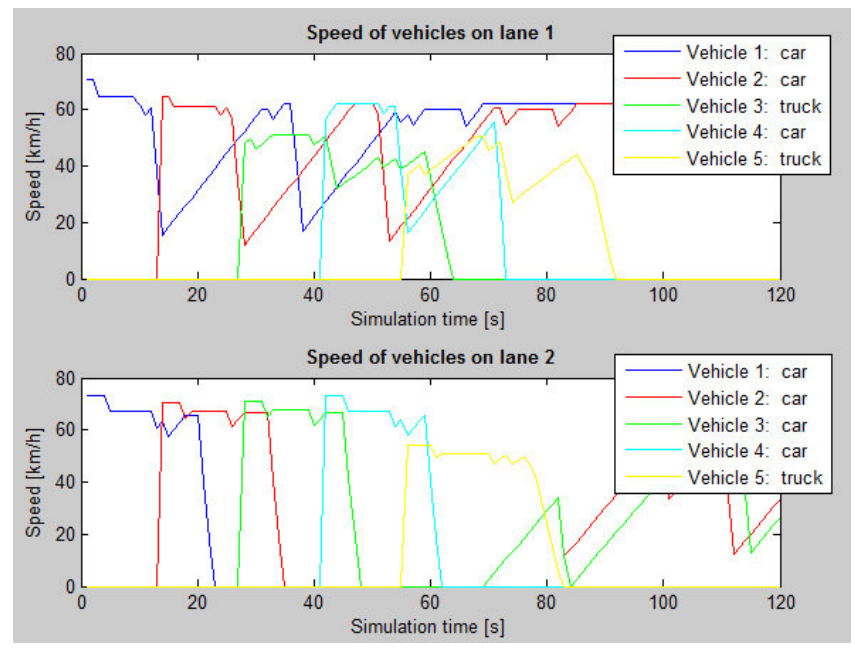

Fig. 8. Vehicle speed plots of the first five vehicles of the simulation with traffic lights in the hazardous area on the Fontanney section. We observe the two huge slow-downs in lane 1 because of the two hairpin bends. The vehicles on lane 2 are stopped by the red lights before the first hairpin bend.

(+96\%), but is only slightly higher than the results of scenario 1 . This is because traffic lights are located outside of the hazardous area. As vehicles stop at a red light, they have a slightly lower speed in scenario 2 than in scenario 1 when they cross the hazardous area.

\subsection{Advantages and limitations of the dynamic approach}

The main advantage of the dynamic approach for risk calculation on roads is a better representation of real traffic regarding the interaction between different vehicles of different types (cars, trucks, coaches). If the traffic on the motorway can be assimilated to a stream composed of vehicles (Treiber, 2010), the traffic on mountain roads strongly depends on interactions between vehicles. This is why the dynamic approach is particularly well suited to winding and/or steep roads, as it is not based on an average vehicle behavior.

The dynamic approach to calculate risk with a microscopic traffic simulator is well designed to analyse in detail the risk on relatively short road sections (up to few kilometres). At regional scale, the risk estimations would be averaged over the entire network (with large parts at no risk) and differences between static and dynamic risks are not so pronounced. Thus, the interest of this method is to analyse hotspots, i.e. strongly hazardous short road sections, and to see for example how the location of traffic lights can increase or reduce the risk.

The present version of the simulator could be improved by developing a 3-D model (to integrate road slope and 3D visibility based on the DEM). Looking forward, we could imagine introducing the simulator as an applet in a GIS.

\subsection{Recommendations}

Despite the simplicity of the model, it highlights some measures that can help risk reduction by keeping the traffic moving as freely as possible. For instance, it shows the importance of optimizing the position of traffic lights relative to hazardous areas; otherwise the risk can easily be multiplied by a factor of two. Speed in hazardous areas must be defined to fluidize traffic as much as possible and to avoid vehicles passing through the section at a reduced speed, which increases the probability of being hit by a natural hazard. Speed on sinuous sections should be chosen to minimize the risk of accidents between vehicles or between a vehicle and a natural hazard; the result of an accident is the stopping of vehicles, which increases drastically the exposure to hazard.

Practically, it is difficult to reduce the risk only by signalization. Field observations have shown that the speed limit is often not respected on mountain roads; local drivers who know the road well often drive much faster. It may be easier to take protective measures such as nets, dams, anchors, etc. Then, the dynamic traffic simulator may help to locate critical areas in terms of traffic. It can be used as a tool to support decision-making for the construction of mitigation measures.

\section{Conclusions}

This new approach for risk assessment on roads with dynamic traffic parameters allows for being slightly more realistic than common methodologies by using only static values and therefore simplifying the risk calculation in complex hazardous situations. As inter-vehicle interactions on mountain roads are important, the integration of these interactions in the model significantly changes the risk estimations. For example, a slow vehicle may generate a vehicle tailback on a sinuous road and the dynamic approach can model this traffic situation and its impact on the final risk.

The traffic simulator on mountain roads developed for this new risk calculation is a simplified kinematic model of a real traffic situation. Even if it can be improved, this first version gives satisfying results regarding the dynamic risk. The simulator highlights the limitations of static risk calculation on winding roads where vehicles move slower than the speed limit or in scenarios with obstacles on the road or traffic lights. Simulations have only required some on-site measurements for calibration with realistic data (e.g. speed). In the future, we can expect that such simulators will provide a simple but effective tool to better assess the risks in complex settings in relation with traffic and to help local experts to make decisions for risk reduction on roads. 


\section{Appendix A}

Pseudo-code of vehicle behaviours.

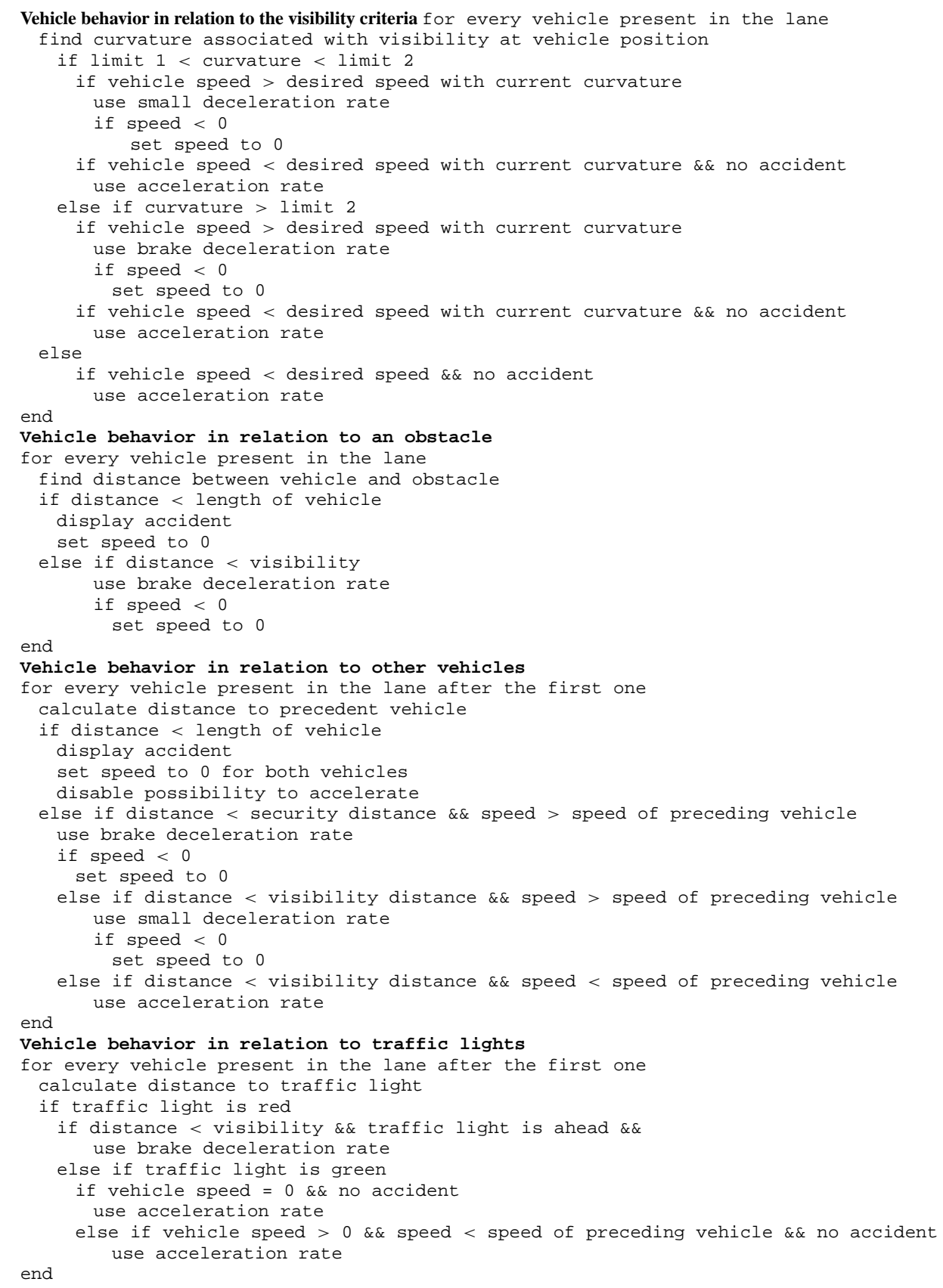

Note that each vehicle can only accelerate, decelerate or break once during a time step.

Vehicle speed is always controlled so that it cannot become negative. 


\section{Appendix B}

Table B1. Simulation risk results of Fontanney section. Three scenarios of dynamic risk: free road (scenario 1) and traffic lights (scenario 2).

\begin{tabular}{lrrr}
\hline $\begin{array}{l}\text { Fontanney } \\
\text { section }\end{array}$ & Lane 1 & Lane 2 & $\begin{array}{r}\text { Sum of } \\
\text { both lanes }\end{array}$ \\
\hline Risk [dead yr ${ }^{-1}$ ] & & & \\
Static risk & 0.029 & 0.029 & 0.057 \\
Dyn. risk, scenario 1 & 0.046 & 0.038 & 0.083 \\
Dyn. risk, scenario 2 & 0.080 & 0.065 & 0.145 \\
\hline Increase relatively to static risk [\%] & & \\
Dyn. risk, scenario 1 & 59 & 31 & 45 \\
Dyn. risk, scenario 2 & 182 & 128 & 155 \\
\hline
\end{tabular}

Table B2. Simulation risk results of Pont-Bourquin section. Three scenarios of dynamic risk: free road (scenario 1) and traffic lights (scenario 2).

\begin{tabular}{lrrr}
\hline $\begin{array}{l}\text { Pont-Bourquin } \\
\text { section }\end{array}$ & Lane 1 & Lane 2 & $\begin{array}{r}\text { Sum of } \\
\text { both lanes }\end{array}$ \\
\hline Risk [dead yr ${ }^{-1}$ ] & 0.007 & 0.007 & 0.013 \\
Static risk & 0.010 & 0.012 & 0.022 \\
Dyn. risk, scenario 1 & 0.012 & 0.014 & 0.025 \\
Dyn. risk, scenario 2 & 52 & 89 & 71 \\
\hline Increase relatively to static risk [\%] & & \\
Dyn. risk, scenario 1 & 79 & 113 & 96 \\
Dyn. risk, scenario 2 & & & \\
\hline
\end{tabular}

Table B3. Simulation risk results of Pillon section. Three scenarios of dynamic risk: free road (scenario 1) and traffic lights (scenario 2).

\begin{tabular}{lrrr}
\hline $\begin{array}{l}\text { Pillon } \\
\text { section }\end{array}$ & Lane 1 & Lane 2 & $\begin{array}{r}\text { Sum of } \\
\text { both lanes }\end{array}$ \\
\hline Risk [dead yr ${ }^{-1}$ ] & & & \\
Static risk & 0.0025 & 0.0025 & 0.005 \\
Dyn. risk, scenario 1 & 0.0034 & 0.0028 & 0.006 \\
Dyn. risk, scenario 2 & 0.0075 & 0.0040 & 0.011 \\
\hline Increase relatively to static risk [\%] & & \\
Dyn. risk, scenario 1 & 38 & 13 & 25 \\
Dyn. risk, scenario 2 & 199 & 60 & 130 \\
\hline
\end{tabular}

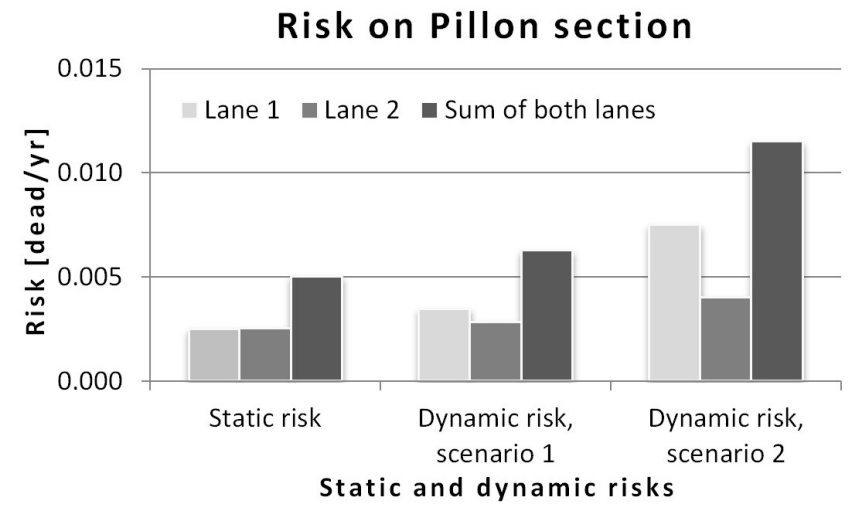

Fig. B1. Risk results on Fontanney section.

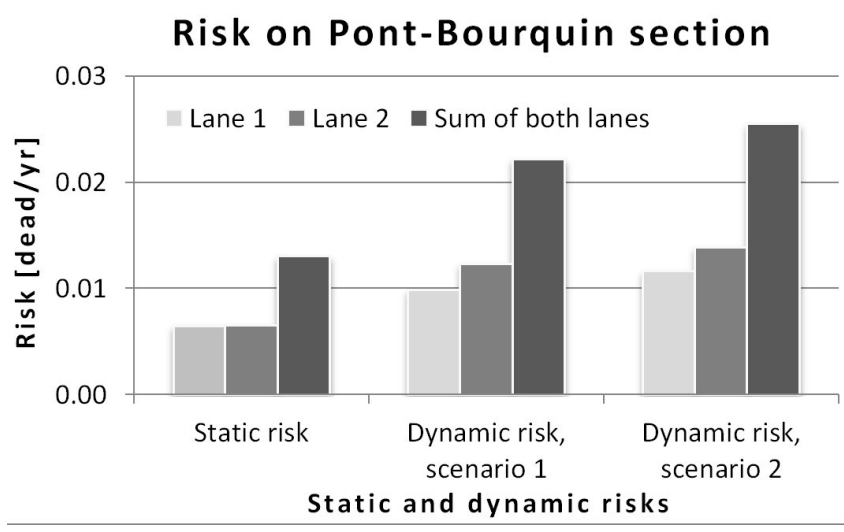

Fig. B2. Risk results on Pont-Bourquin section.

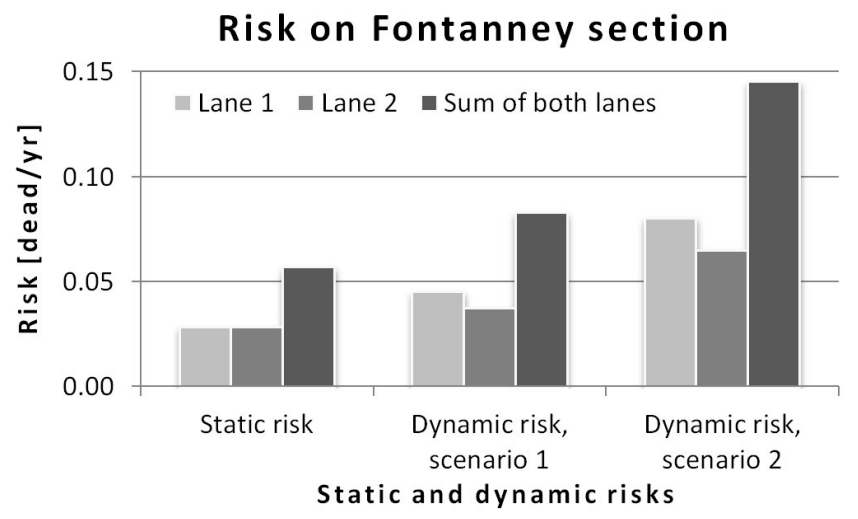

Fig. B3. Risk results on Pillon section. 
Acknowledgements. The authors thank Céline Longchamp and Simon Hiscox for her precious help and her constructive remarks to improve the quality of the manuscript. Grateful thanks also to the two reviewers for helpful comments and valuable guidance on an earlier version of the manuscript.

Edited by: P. Reichenbach

Reviewed by: G. M. Stock and two anonymous referees

\section{References}

Al-Homoud, A. S., Tal, A. B., and Taqieddin, S. A.: A comparative study of slope stability methods and mitigative design of a highway embankment landslide with a potential for deep seated sliding, Eng. Geol., 47, 157-173, 1997.

ASTRA: Annual and monthly results, available at: http://www.astra.admin.ch/verkehrsdaten/00299/00301/05582/ index.html?lang=en (last access: 8 November 2012), 2012.

Baillifard, F., Jaboyedoff, M., and Sartori, M.: Rockfall hazard mapping along a mountainous road in Switzerland using a GIS-based parameter rating approach, Nat. Hazards Earth Syst. Sci., 3, 435442, doi:10.5194/nhess-3-435-2003, 2003.

Barceló, J. (Ed.): Fundamentals of Traffic Simulation, International Series in Operations Research \& Management Science, 145, Springer, New York, 460 pp., 2010.

Borter, P.: Risikoanalyse bei gravitativen Naturgefahren: Methode, Bundesamt für Umwelt, Wald und Landschaft, 115 pp., available at: http://www.bafu.admin.ch/publikationen/publikation/00131/ index.html?lang=de, 1999.

Bründl, M.: Guide du concept du risque, Plateforme nationale "Dangers Naturels" PLANAT, 61 pp., available at: http://www. planat.ch/fileadmin/PLANAT/planat_pdf/alle_2012/2006-2010/ PLANAT_2009_-_Guide_du_concept_de_risque.pdf, 2009.

Budetta, P.: Assessment of rockfall risk along roads, Nat. Hazards Earth Syst. Sci., 4, 71-81, doi:10.5194/nhess-4-71-2004, 2004.

Bunce, C. M., Cruden, D. M., and Morgenstern, N. R.: Assessment of the hazard from rock fall on a highways, Can. Geotech. J., 24, 344-356, 1997.

Cajos, J., Trocme-Maillard, M., Huber, M., Arnold, P., Vollmer, U., Sandri, A., Raetzo, H. Dorren, L. K. A., Egli, T., Eberli, J., Knuchel, R., Kienholz, H., Donzel, M., Utelli, H. H., and Perren, B.: Risk concept for natural hazards on national roads, Federal Roads Office, 108 pp., available at: http://www.astra.admin.ch/dienstleistungen/00129/, 2009.

Cameron, G. D. B. and Duncan, D. I. D.: PARAMICS - Parallel Microscopic Simulation of Road Traffic, J. Supercomput., 10, 25-53, 1996.

Canton de Vaud: Trafic journalier moyen (TJM), available at: http://www.vd.ch/themes/mobilite/routes/le-reseau-routier/ trafic-journalier-moyen-tjm/ (last access: 8 November 2012), 2012.

Chiu, Y. C., Zheng, H., Villalobos, J. A., Peacock, and Henk, R.: Evaluating Regional Control- Flow and Phased Evacuation Strategies for Texas Using a Large-Scale Dynamic Traffic simulation and Assignment Approach, J. Homeland Security Emergency Manage., 5, 34, doi:10.2202/1547-7355.1409, 2008.

Collins, T. K.: Debris flows caused by failure of fill slopes: early detection, warning, and loss prevention, Landslides, 5, 107-120, 2008.
Cruden, D. M. and Varnes D. J.: Landslide types and processes, in: Special Report 247, Landslides: Investigation and Mitigation, edited by: Turner, A. K. and Shuster R. L., Transportation Ressearch Board, Spec Rep 247, Washington DC, 36-75, 1996.

Dorren, L. K. A., Sandri, A., Raetzo, H., and Arnold, P.: Landslide risk mapping for the entire Swiss national road network, in: Landslide Processes: from geomorphologic mapping to dynamic modeling, Strasbourg, France, 6-7 February 2009, 277281, 2009.

Du, Z. P. and Nicholson, A.: Degradable transportation systems: Sensitivity and Reliability Analysis, Transportation Res. B, 31, 225-237, 1997.

Einstein, H. H.: Special lecture: landslide risk assessment procedure, in: Proceeding, 5th International Symposium on Landslides, edited by: Bonnard, C., Lausanne, Vol. 2, Rothherdam: A. A. Balkema, 1075-1090, 1988.

FEDRO: Risk concept for natural hazards on national roads, Federal Roads Office, 108 pp., available at http://www.astra.admin.ch/dienstleistungen/00129/, 2009.

FEDRO: Comptage suisse automatique de la circulation routière (CSACR), available at: http://www.portal-stat.admin.ch/sasvz/ index.html (last access: 8 November 2012), 2012.

Fell, R., Ho, K. K. S., Lacasse, S., and Leroi, E.: A framework for landslide risk assessment and management, in: Landslide Risk Management, edited by: Hungr, O., Fell, R., Couture, R. and Eberhardt, E., Proceedings of the International Conference on Landslide Risk Management in Vancouver, Canada, 31 May - 3 June 2005, 3-25, 2005.

Guemache, M. A., Chatelain, J.-L., Machane, D., Benahmed, S., and Djadia, L.: Failure of landslide stabilization measures: The Sidi Rached viaduct case (Constantine, Algeria), J. African Earth Sci., 59, 349-358, 2011.

Hungr, O., Evans, S. G., and Hazzard, J.: Magnitude and frequency of rock falls and rock slides along the main transportation corridors of southwestern British Columbia, Can. Geotech. J., 36, 227-238, 1999.

Hungr, O., Leroueil, S., and Picarelli, L.: Varnes classification of landslide types, an update, in: Landslides and Engineered Slopes, edited by: Eberhardt, C., Froese, E., Turner, A. K., and Leroueil, S., London, 47-58, 2012.

Jha, M., Moore, K., and Pashaie, B.: Emergency Evacuation Planning with microscopic Traffic Simulation, Transportation Research Record, J. Transport. Res. Board, 1886, 40-48, 2004.

Kerner, B. S.: Introduction to Modern Traffic Flow Theory and Control: The Long Road to Three-Phase Traffic Theory, Springer, 265 pp., 2004.

Khisty, J. and Lall, K.: Transportation Engineering: An Introduction, Prentice Hall, 813 pp., 2003.

Li, Z. and Duan, H.: Acceleration estimation method and sliding mode control design for car-following distance control, Procedia Eng., 15, 1176-1180, 2011.

MASSA: Documents de présentation de l'Action 1, Les méthodes:, available at: http://massa.geoazur.eu/action1/Doc_present. php (last access: 31 January 2012), 2010.

McShane, W., Roess, R., and Prassas E.: Traffic Engineering, Prentice Hall, 714 pp., 1998.

Michoud, C., Derron, M.-H., Horton, P., Jaboyedoff, M., Baillifard, F.-J., Loye, A., Nicolet, P., Pedrazzini, A., and Queyrel, A.: Rockfall hazard and risk assessments along roads at a regional 
scale: example in Swiss Alps, Nat. Hazards Earth Syst. Sci., 12, 615-629, doi:10.5194/nhess-12-615-2012, 2012.

Miller, A.: A Queuing Model for Road Traffic Flow, J. Roy. Stat. Society, Ser. B, 23 64-90, 1960.

Moshe, B.-A., Haris, N. K., Tomer, T., Qi, Y., and Charisma, F. C., Constantinos, A., and Ramachandran, B.: Traffic Simulation with MITSIMLab, in: Fundamentals of Traffic Simulation, edited by: Barceló, J., International Series in Operations Research \& Management Science, 145, 233-268, 2010.

Nicholson, A. and Du, Z. P., Degradable transportation systems: an Integrated Equilibrium Model, Transport. Res. B, 31, 209-223, 1997.

Peng, G. H., Cai, X. H., Liu, C. Q., Cao, B. F., and Tuo, M. X.: Optimal velocity difference model for a car-following theory, Phys. Lett. A, 375, 3973-3977, 2011.

Pierson, L. A., Davis, S. A., and Van Vickle, R.: Rockfall Hazard Rating System - Implementation Manual, Federal Highway Administration (FHWA), Report FHWA-OR-EG-90-01, FHWA, US Dep. of Transp., 1990.

Rajat, J. and MacGregor-Smith, J.: Modeling Vehicular Traffic Flow using M/G/C/C State Dependent Queueing Models, Transport. Sci., 31, 324-336, 1997.
Roberds, W.: Estimating temporal and spatial variability and vulnerability, in: Landslide Risk Management, edited by: Hungr, O., Fell, R., Couture, R. and Eberhardt, E., Proceedings of the International Conference on Landslide Risk Management in Vancouver, Canada, 31 May-3 June 2005, 129-157, 2005.

Salcedo, D. A.: Behavior of a landslide prior to inducing a viaduct failure, Caracas-La Guaira highway, Venezuela, Eng. Geol., 109, 16-30, 2009.

Schönhof, M. and Helbing, D.: Criticism of three-phase traffic theory, Transport. Res. Part B, 43, 784-797, 2009.

Treiber, M. and Kesting, A.: Verkehrsdynamik und -simulation, Springer, 368 pp., 2010.

Wilhelm, C.: Wirtschaftlichkeit im Lawinenschutz, Methodik une Erhebungen zur Beurteilung von Schutzmassnahmen mittels quantitativer Risikoanalyse und ökonomischer Bewertung, Eidgenössisches Institut für Schnee- und Lawinenforschung, 1997.

Yang, Q., Koutsopoulos, H. N., and Ben-Akiva, M.: A simulation laboratory for evaluating dynamic traffic management systems, Transport Res. Rec., 1710, 122-130, 2000.

Yu, S., Liu, Q., and Li, X.: Full velocity difference and acceleration model for a car-following theory, Commun. Nonlinear Sci. Numer. Simulat., 18, 1229-1234, 2013. 